\title{
Conservación de un área protegida con uso recreativo: ¿Se puede lograr que los visitantes dejen menos basura?
}

\author{
Ana M. Cingolani ${ }^{1,2 \varpi ; ~ I v a ́ n ~ B a r b e r a ́ ~}{ }^{2}$; Daniel Renison ${ }^{3} \&$ Fernando R. Barri ${ }^{2,4}$ \\ 1. Instituto Multidisciplinario de Biología Vegetal, Conicet- Universidad Nacional de Córdoba, Córdoba, Argentina. 2. \\ Facultad de Ciencias Exactas, Físicas y Naturales, Universidad Nacional de Córdoba, Córdoba, Argentina. 3. Instituto \\ de Investigaciones Biológicas y Tecnológicas, Centro de Ecología y Recursos Naturales Renovables (Conicet- Universidad \\ Nacional de Córdoba), Córdoba, Argentina. 4. Instituto de Diversidad y Ecología Animal, Conicet-Universidad Nacional de \\ Córdoba, Córdoba, Argentina.
}

\begin{abstract}
Resumen. Uno de los problemas de las áreas protegidas en zonas de afluencia masiva es la acumulación de basura abandonada por algunos visitantes. Evaluamos, en playas ribereñas de un área protegida de las sierras de Córdoba, si es posible reducir la basura abandonada por los visitantes utilizando herramientas de la interpretación ambiental. La técnica utilizada consistió en el pedido personalizado al visitante de no dejar basura, acompañado del ejemplo de juntar la basura abandonada en la playa. Realizamos el estudio en nueve playas, haciendo un total de 72 observaciones (repartidas en 10 fechas), consistentes en tres recorridos: antes, durante y después de la hora de máxima afluencia de visitantes. En el recorrido anterior a la estadía de los visitantes limpiamos las playas. Durante la hora de máxima afluencia contamos los visitantes, y realizamos la intervención, consistente en el pedido personalizado y el ejemplo, aproximadamente en la mitad de las playas ( $N=37$ y $N=35$ observaciones con y sin intervención respectivamente). Después de la estadía de los visitantes, a la mañana siguiente, para cada playa estimamos la cantidad de basura, que varió entre 0.007 a 32.5 g.visitante ${ }^{-1}$.día ${ }^{-1}$. En siete de las nueve playas, la cantidad de basura por visitante fue más baja para las fechas en las cuales se realizó la intervención que para las fechas en las cuales no se realizó, pero las diferencias no fueron significativas $(P=0.42)$. En cambio, encontramos diferencias significativas entre playas $(P<0.05)$, detectando por medio de correlaciones que en las playas más chicas los visitantes dejaron menos basura que en las playas más grandes $(R=0.74 ; P=0.022)$. Concluimos que si bien el efecto del pedido personalizado no pudo ser demostrado estadísticamente, las tendencias encontradas son alentadoras, y es necesario seguir investigando.
\end{abstract}

[Palabras clave: balnearios, conservación, interpretación ambiental, mensaje persuasivo, residuos, turismo]

\begin{abstract}
Conservation of a protected area with recreational use: is it possible to persuade visitors to produce less litter? Littering by visitors is a problem in many highly visited protected areas. We evaluated, in river beaches within a protected area in the mountains of Córdoba, Argentina, whether it is possible to reduce visitors' littering using environmental interpretation tools. The interpretation tool consisted in a personalized request to the visitors asking them not to litter while picking up the litter already left on the beach. We conducted the study at nine beaches, making a total of 72 observations (spread over 10 dates). Each observation consisted of three rounds: before, during and after the time of the day with maximum influx of visitors. In the round before we cleaned the beaches. During peak visitation we counted visitors and applied the intervention, which consisted in the personalized request and the example, in about half of the beaches ( $N=37$ and $N=35$ with and without intervention respectively). After the peak visitation, the next morning we estimated the amount of litter per beach, which ranged from 0.007 to 32.5 g.visitor ${ }^{-1}$.day ${ }^{-1}$. In seven out of the nine beaches, the amount of litter per visitor was lower for the dates on which the personalized request was applied, but the differences were not significant $(P=0.42)$. Instead, we found significant differences among beaches $(P \leq 0.05)$, and a significant positive correlation between beach size and the amount of litter per visitor $(R=0.74, P \leq 0.05)$. We concluded that despite we couldn't demonstrate statistically the effect of the personalized request, the observed trends are encouraging, and it is necessary to continue the research
\end{abstract}

[Keywords: beaches, conservation, environmental interpretation, persuasive message, litter, tourism]

\section{INTRODUCCIÓN}

El acelerado avance de la urbanización está causando una retracción importante de las áreas naturales, y a veces ecosistemas enteros están amenazados por esta causa (McKinney 2002). En estas circunstancias resulta importante proteger algunos fragmentos silvestres dentro de las zonas urbanas, tanto con el objetivo de conservar la biodiversidad, como el de mantener los servicios ecosistémicos que las áreas naturales pueden brindar a los habitantes urbanos (McKinney 2002; Millenium Ecosystem Assessment 2005; Juniper 2013). En tal sentido tiene particular importancia conservar las márgenes de ríos y arroyos en estado natural. De este modo, 
contribuyen a mantener la calidad del agua, entre otros beneficios, al actuar como áreas de amortiguamiento del impacto producido en otras partes de la cuenca (Karr 1991; Sliva \& Willams 2001; Mayer et al. 2007).

En lugares turísticos, sobre todo aquellos utilizados como balnearios, conservar la calidad del agua y la belleza paisajística es prioritario; y al mismo tiempo la intensa presión de urbanización atenta contra ambos objetivos (Morley \& Karr 2002; McKinney 2002; Dadon 2002; Barragán-Muñoz et al. 2010). Por ello, la creación de áreas protegidas en las márgenes de los cuerpos de agua puede ser una medida muy eficaz para el manejo de estos lugares (Abell et al. 2007). Uno de los factores que pueden amenazar a un área protegida en zonas urbanas o en proceso de urbanización es la basura. Este problema es particularmente importante en las áreas de afluencia masiva, ya que la cantidad de basura encontrada en un lugar es proporcional al número de visitantes que recibe (Santos et al. 2005; dos Santos et al. 2008; Seco Pon \& Becherucci 2012). El efecto negativo más evidente de la basura es la contaminación visual, pero también puede producir contaminación del suelo, de los cursos de agua, o perjudicar a la fauna, a veces causando incluso la muerte de animales (Santos et al. 2005; Brown et al. 2010). Por otro lado, la vegetación en estado natural en combinación con la basura es percibida como descuido o dejadez, lo cual en zonas urbanas aumenta la presión social por parquizar o privatizar el área (Pers. obs.). Asimismo, en el caso de los balnearios, la basura abandonada en lugares inadecuados atenta directamente contra la calidad del recurso turístico, pudiendo, en conjunto con otros factores, afectar negativamente a la economía local (Ballance et al. 2000; Dadón et al. 2002; Barragán-Muñoz et al. 2003; Santos et al. 2005).

Para evitar los inconvenientes que genera la basura es importante que las áreas naturales permanezcan limpias, y una manera de contribuir con este objetivo es incentivar a los visitantes que aún no lo hacen para que arrojen sus residuos en los lugares adecuados. En tal sentido, la interpretación ambiental, entendida como una actividad educativa orientada a revelar significados y relaciones a través de experiencias directas y medios ilustrativos (Ham 1992) puede ser de gran ayuda. La comunicación persuasiva es una herramienta de la interpretación ambiental que puede ser muy útil para alcanzar el objetivo de mantener las áreas limpias (Ham 1992; Marion \& Reid 2007; Brown et al. 2010). Los mensajes persuasivos, ya sea a través de pedidos personalizados, carteles o folletos, buscan comunicar la razón detrás de las normas, sin transmitir una amenaza de sanción ante el no-cumplimiento, y muchas veces han mostrado ser igual o más efectivos que los mensajes que sí transmiten una amenaza de sanción (Duncan \& Martin 2002). En el caso de la basura, los mensajes persuasivos permiten estimular la sensibilidad de los visitantes en relación al problema, logrando así una buena predisposición a comportarse adecuadamente (Ham 1992; Orams 1997; Cialdini 2003). A raíz de ello se puede conseguir una reducción efectiva de la basura abandonada en lugares inadecuados (Brown et al. 2010). Si los mensajes persuasivos se combinan con mensajes demostrativos señalando a los visitantes de un determinado lugar que la mayor parte de las personas respetan las normas, se pueden lograr resultados aún mejores (Cialdini 2003; Keizer et al. 2008, 2013). En el caso de la basura, los mensajes demostrativos pueden consistir en señales débiles, como simplemente mantener el lugar limpio, o señales más fuertes como el mostrar personas llevándose incluso la basura que dejaron otras personas. En estos últimos casos la efectividad de los mensajes es máxima (Keizer et al. 2013). Por estas razones, una de las técnicas de interpretación ambiental que se utiliza para lograr objetivos comportamentales es combinar un pedido de manera verbal con brindar el ejemplo (Brown et al. 2010).

En los países latinoamericanos, incluyendo Argentina, hay numerosos estudios que indican que el problema de la basura en áreas protegidas, urbanizaciones y balnearios es grave y de alta prioridad (e.g. Santos et al. 2005; dos Santos et al. 2008; Bravo et al. 2009; Thiel et al. 2011; Seco-Pon \& Becherucci 2012; Easteman et al. 2013). En muchos de los mencionados estudios se propone la educación o interpretación ambiental como forma de generar un cambio de comportamiento, pero la efectividad de este tipo de actividades no se ha evaluado experimentalmente en estos países. Por ello nos propusimos evaluar la efectividad de los mensajes persuasivos y demostrativos en una pequeña reserva serrana que incluye balnearios ribereños con una alta afluencia de visitantes en el período estival. Específicamente, el objetivo del trabajo fue analizar si el pedido personalizado, combinado con el ejemplo dado por un voluntario, produce una reducción en la 
cantidad de basura que es abandonada en la playa por los visitantes.

\section{MÉTOdos}

\section{Área de estudio}

El estudio se realizó en la Reserva Ecológica y Recreativa Cuesta Blanca, en la Comuna del mismo nombre ( $31^{\circ} 28^{\prime} 59^{\prime \prime} \mathrm{S}, 64^{\circ} 34^{\prime} 34^{\prime \prime} \mathrm{O}$, Córdoba, Argentina). La reserva abarca 14 ha en las márgenes del río San Antonio, uno de los principales afluentes del lago San Roque. La misma cuenta con cartelería que la identifica, así como con señales indicando cómo debe ser el comportamiento de los visitantes, incluyendo el pedido de arrojar la basura en lugares habilitados. Fue creada por el gobierno comunal en el año 2009 con los objetivos de preservar un área de amortiguamiento ribereña, contribuyendo así a conservar la calidad del agua, recuperar el bosque serrano nativo, evitar la erosión de los suelos, proteger especies de flora y fauna, y crear un espacio educativo y recreativo (Cingolani 2013). El área está dominada por bosque chaqueño serrano en distintos estados sucesionales, principalmente matorral, con algunos sectores muy invadidos por especies exóticas (Giorgis et al. 2011). Este bosque forma una franja de ancho variable (entre 5 y $80 \mathrm{~m}$ ) en las márgenes del río, y las zonas con vegetación están combinadas con áreas rocosas y playas de arena utilizadas por los visitantes. En la época estival, el número de visitantes es máximo, y consiste principalmente de grupos familiares, parejas y grupos de jóvenes que provienen de localidades cercanas, ciudad de Córdoba y diferentes ciudades del país (Obs. Pers.). Una de las particularidades de esta pequeña reserva es que se ubica dentro de una cuenca que es considerada como de alto riesgo socio-ecológico, dados los serios problemas ambientales que enfrenta, la poca participación de una gran parte de los organismos gubernamentales en la búsqueda de soluciones a los problemas ambientales, y la conducta fragmentada o no-cooperativa de los distintos actores sociales que intervienen sobre la cuenca (Berardo 2014).

\section{Diseño de muestreo y toma de datos}

Seleccionamos un total de 10 playas en el río San Antonio, nueve de ellas en la zona de Reserva Cuesta Blanca, y una en la localidad vecina (Icho Cruz), fuera de la reserva pero ubicada a pocos metros de la misma. Las playas se extienden a lo largo de 1.22 $\mathrm{km}$ en ambas márgenes del río y el acceso a todas ellas es peatonal. En general están separadas entre sí por zonas más rocosas o vegetadas, menos utilizadas por los visitantes. Delimitamos las playas sobre una imagen impresa de Google-Earth, constatamos los límites en el terreno, y luego las digitalizamos y calculamos la superficie de cada una, que varió entre 213 y $1047 \mathrm{~m}^{2}$ (Tabla 1).

Entre enero y marzo de 2013, época del año de mayor afluencia de visitantes a la reserva, estudiamos las playas en un total de 10 fechas (los domingos 13, 20 y 27 de enero, 3, 10 y 24 de febrero, 3, 10 y 24 de marzo, y el sábado 30 de marzo). En cada fecha recorrimos todas las playas tres veces: antes, durante y después de la estadía de los visitantes (con algunas excepciones, ver más adelante). Antes de la llegada de los visitantes juntamos toda la basura detectada para comenzar el día con las playas limpias. Durante el horario de máxima afluencia de visitantes (entre las 17 y las 19 hasta mitad de febrero, y luego entre las 16 y las 18) hicimos otro recorrido, en el cual contamos el número de visitantes presentes dentro de los límites de cada playa y realizamos una intervención en la mitad de ellas. Después de la estadía de los visitantes, temprano por la mañana del día siguiente, colectamos la basura que había quedado en cada playa. La intervención, realizada en la mitad de las playas, consistió en la visita de una misma persona (Iván Barberá), que actuaba como guardambiente voluntario en la localidad y estaba identificado como tal. Durante la visita, el guardambiente juntaba la basura que estaba abandonada en la playa, a modo de brindar un ejemplo a los visitantes, y la colocaba en bolsas de polietileno que luego se rotulaban con el nombre de cada playa. Además, el guardambiente se acercaba a todos los visitantes o grupos que se encontraban dentro de los límites de la playa y conversaba con cada uno de forma personalizada. Explicaba que él era voluntario, y solicitaba a las personas que al irse, llevasen la basura a los cestos (situados a distancias de entre 26 a $204 \mathrm{~m}$ de las playas), incluyendo las

Tabla 1. Las nueve playas ${ }^{1}$ utilizadas para el análisis de la cantidad de basura por visitante, la superficie, y la cantidad de observaciones completas sin y con intervención (pedido personalizado + ejemplo de juntar la basura abandonada) respectivamente.

Table 1. The nine beaches ${ }^{1}$ used for the analysis of the amount of litter per visitor, their area, and the number of complete observations without and with intervention (personalized request + demonstration of picking up the deposited litter) respectively.

\begin{tabular}{lcccc}
\hline $\mathrm{N}^{\circ}$ & Nombre & $\begin{array}{c}\text { Superficie } \\
\left(\mathrm{m}^{2}\right)\end{array}$ & $\begin{array}{c}\text { Sin } \\
\text { intervención }\end{array}$ & $\begin{array}{c}\text { Con } \\
\text { intervención }\end{array}$ \\
\hline A & Curva & 734 & 4 & 4 \\
B & Saltito & 745 & 5 & 3 \\
C & Playita & 772 & 3 & 4 \\
D & Piedra del Indio & 683 & 5 & 4 \\
E & Ruiseñor & 1047 & 4 & 4 \\
F & Guindillo & 439 & 4 & 5 \\
G & Comuna & 520 & 3 & 3 \\
H & Escaladores & 331 & 4 & 5 \\
I & Ballena & 213 & 3 & 5 \\
& TOTAL & 5739 & 35 & 37 \\
\hline
\end{tabular}

'La playa J (Icho Cruz) se descartó de los análisis de datos de basura por visitante, por tener una sola observación con intervención, pero se mantuvo en el análisis de la densidad de personas que concurren a las playas.

${ }^{1}$ Beach J (Icho Cruz) was discarded from the analysis of visitors' littering because it had only one observation with intervention, but it was maintained in the analysis of visitors' density. 
colillas de cigarrillo, expresando amablemente la importancia de mantener limpio el lugar. En algunos casos, cuando había disponibilidad, brindaba a los visitantes un folleto elaborado por el gobierno comunal, con algunas explicaciones y pautas de respeto a la naturaleza (Cuesta Blanca 2011). El conjunto de playas en las cuales se realizó la intervención y el orden en que se recorrieron las playas fue variable entre fechas. Sin embargo, procuramos que en cada fecha de observación la mitad de las playas recibiera la intervención y la otra mitad no, y que al final del estudio todas las playas tuviesen un número similar de fechas con y sin intervención. Después de la estadía de los visitantes, para cada fecha pesamos la basura colectada en cada una de las 10 playas, agregando cuando correspondía la basura juntada a modo de ejemplo el día anterior durante el horario de máxima afluencia de visitantes.

En total hicimos 95 observaciones (10 playas $\mathrm{x}$ 10 días, descartando cinco observaciones en las cuales no había visitantes en el momento de la intervención). De estas 95 observaciones, en 17 no pudimos hacer correctamente la limpieza previa y las descartamos del análisis de la cantidad de basura abandonada. Debido a ello, tuvimos que descartar también una de las playas, por haber quedado con una sola observación en la cual se había aplicado la intervención (pedido personalizado + ejemplo). De este modo, quedaron un total de 72 observaciones completas para la comparación entre situaciones con y sin intervención, Tabla 1).

\section{Análisis de datos}

Para cada fecha estimamos la densidad de visitantes por playa en el horario de máxima afluencia como el cociente entre la cantidad de personas contadas y la superficie de cada playa. Dado que la densidad podría influir sobre el comportamiento individual de arrojar basura, nos interesó describir su variación en el tiempo y espacio. Para comparar el patrón de densidades entre fechas y entre playas hicimos un ANOVA a dos factores, utilizando las 100 observaciones con recuento de personas (10 fechas x 10 playas, incluyendo las cinco observaciones con ausencia de visitantes).

Para cada fecha y playa, considerando sólo las 72 observaciones completas (Tabla 1), estimamos la cantidad promedio de basura abandonada por visitante (g.visitante ${ }^{-1}$.día $\mathrm{a}^{-1}$ ) como el cociente entre el peso de la basura colectada y la cantidad de visitantes evaluada en la hora de máxima afluencia. Este cociente sobreestima levemente la cantidad de basura por visitante, ya que el recuento de personas se hizo en un único momento, durante el período de máxima afluencia, mientras que una proporción de la basura colectada pudo haber sido abandonada por personas que visitaron la playa durante el día pero no estaban presentes en el momento de máxima afluencia. Dado que esta proporción es baja (Obs. Pers.), consideramos que no es necesario aplicar ninguna corrección para hacer el cálculo.

Para cada playa y fecha, transformamos el valor de la cantidad de basura por visitante a su logaritmo natural (previo a lo cual sumamos 1 para evitar valores negativos), y utilizamos esta variable transformada como variable de respuesta en un modelo lineal general. Hicimos la transformación para normalizar los datos, ya que había algunos valores excesivamente altos (debidos principalmente a botellas de vidrio). Como variables explicativas, consideramos dos variables categóricas: "intervención" (con y sin) y "playa" (9 categorías, Tabla 1), y una variable continua: "densidad de visitantes". Una vez realizado el análisis de datos, calculamos la potencia observada para cada una de las dos variables categóricas analizadas.

Finalmente, dado que el factor "playa" resultó importante en su efecto sobre la cantidad de basura por visitante (ver resultados), para detectar posibles factores explicativos de las diferencias entre playas calculamos un único promedio para cada playa de la variable de respuesta transformada (ln de la cantidad de basura por visitante). Realizamos correlaciones de Pearson entre este promedio y el tamaño de la playa $\left(\mathrm{m}^{2}\right)$, la distancia a la parada de colectivo más cercana $(\mathrm{m})$, y al contenedor de basura más cercano $(\mathrm{m})$.

\section{Resultados}

La cantidad de visitantes varió desde un máximo de 780 personas para el 13 de enero de 2013 (densidad media de 13.9 personas por 100 $\mathrm{m}^{2}$ ), y un mínimo de 42 personas el 3 de marzo de 2013 (densidad media de 0.8 personas por $100 \mathrm{~m}^{2}$ ). Estas diferencias entre fechas en la densidad de visitantes por playa fueron estadísticamente significativas $(F=19.47, P<$ 0.001, Figura 1). La densidad de visitantes también varió significativamente entre playas, indicando que por unidad de superficie, algunas playas fueron sistemáticamente más concurridas que otras $(F=4.85, P<0.001)$. La playa I ("Ballena") fue la más visitada, con una densidad media de 10.33 personas por cada $100 \mathrm{~m}^{2}$, mientras que la playa B ("Saltito") la menos visitada, con una densidad media de 2.74 personas por cada $100 \mathrm{~m}^{2}$ (Figura 1).

La cantidad de basura abandonada varió entre 0.007 g.visitante ${ }^{-1} \cdot$ día $^{-1}$ (30 de marzo de 2013, para la playa $H$, "Escaladores") y 32.5 g.visitante ${ }^{-1}$.día ${ }^{-1}$ (10 de marzo de 2013, para la playa C, "Playita"), con un promedio de 4.03 g.visitante ${ }^{-1}$.día ${ }^{-1}$. La mayor parte de la basura consistió en botellas de plástico, $\mathrm{u}$ ocasionalmente de vidrio, envoltorios de comida y colillas de cigarrillo. Al comprar la cantidad de basura por visitante entre playas 


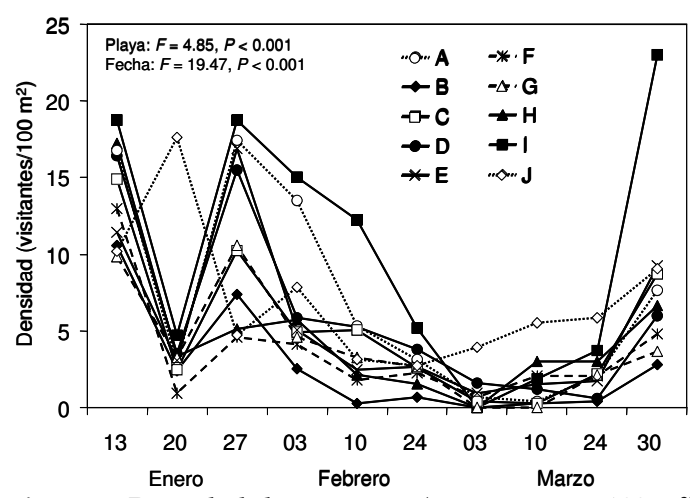

Figura 1. Densidad de visitantes (visitantes por $100 \mathrm{~m}^{2}$ ) en las 10 playas seleccionadas a lo largo de 10 fechas estudiadas.

Figure 1. Visitor density (visitors per $100 \mathrm{~m}^{2}$ ) in the 10 selected beaches along the 10 study dates.

y entre fechas con y sin intervención, sólo el factor playa mostró diferencias significativas $(F=2.68, P=0.014)$. Estas diferencias fueron de casi un orden de magnitud entre la playa más limpia y la más sucia (Figuras 2 y 3). La densidad de visitantes y las interacciones entre factores no fueron significativas, y se descartaron del modelo. Si bien el factor "intervención" no fue significativo, en siete de las nueve playas la basura por visitante fue menor cuando éstos habían recibido el pedido personalizado y observado el ejemplo (Figura 2). La potencia observada para el factor "playa" fue de 0.89 , y para el factor "intervención" de 0.13 . Esto último indica altas probabilidades de que haya diferencias

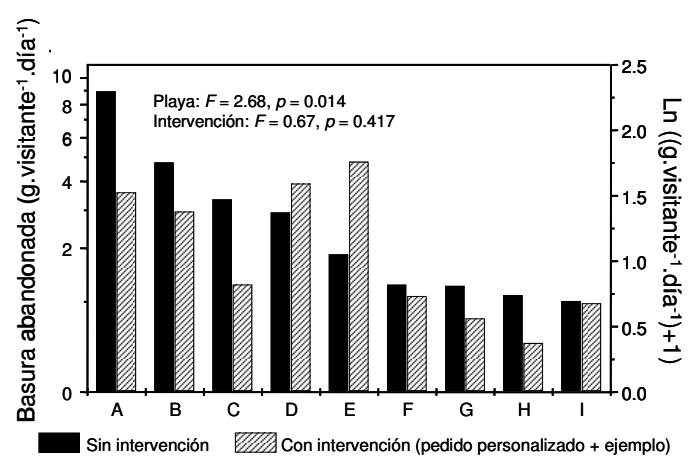

Figura 2. Cantidad promedio de basura por visitante y por día en las nueve playas analizadas, para las fechas con y sin intervención. En la escala de la izquierda se indican los valores en las unidades originales de medición, y en la escala de la derecha se indican los promedios de los valores transformados (tal como se usaron en los análisis estadísticos).

Figure 2. Average amount of litter per visitor and day in the nine study beaches, for the dates with and without intervention. In the left scale we show the values in the original units, and in the right scale we show the averages of $\log$ transformed values (as used in the statistical analyses). significativas entre situaciones con y sin intervención que no fueron detectadas debido a la gran variabilidad de los datos.

Cuando analizamos los posibles factores explicativos de las diferencias entre playas, el área de la playa fue la única variable que tuvo una correlación significativa con la cantidad de basura por visitante $(R=0.74, P=0.022)$. En las playas más grandes, se dejó más basura por visitante que en las playas más chicas (Figura 3). Las otras dos variables no mostraron una correlación significativa con la cantidad de basura dejada por persona $(R=-0.15, P=0.694$ para la distancia a la parada de colectivo más cercana, y $R=0.06, P=0.885$ para la distancia al contenedor de basura más cercano).

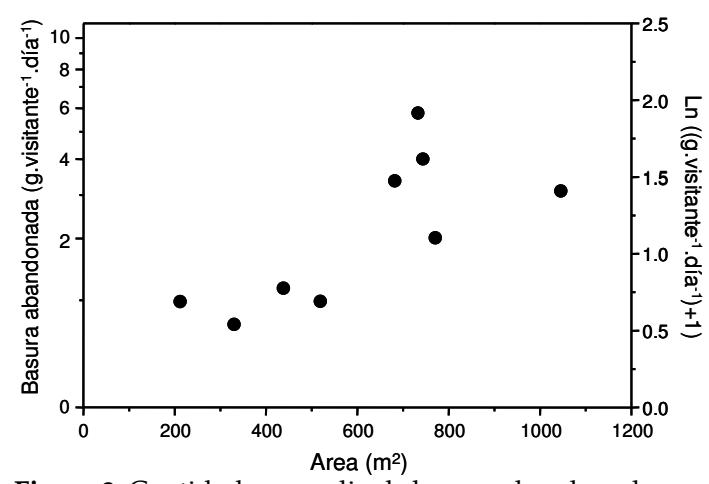

Figura 3. Cantidad promedio de basura abandonada por visitante en función del área de la playa, para las nueve playas analizadas. En la escala de la izquierda se indican los valores en las unidades originales de medición, y en la escala de la derecha se indican los promedios de los valores transformados (tal como se usaron en los análisis estadísticos).

Figure 3. Average amount of litter per visitor as a function of the beach size for nine study beaches. In the left scale we show the values in the original units, and in the right scale we show the averages of log transformed values (as used in the statistical analyses).

\section{DisCUSIÓN}

Las playas fueron muy heterogéneas en la cantidad de basura abandonada por los visitantes. Esta heterogeneidad estuvo explicada en parte por el tamaño de la playa. En promedio, los visitantes que concurrieron a las playas más grandes abandonaron más basura por persona que los visitantes que concurrieron a las playas más pequeñas. Se podría pensar que en las playas grandes, con mayor cantidad de visitantes, la probabilidad de que alguno de ellos abandone basura es mayor que en las playas chicas. Dado que el comportamiento de las personas suele estar regido por la acción que realizan sus pares en el lugar (Cialdini 2003; Páramo 2010; Keizer 
et al. 2008, 2013), esto podría desencadenar el comportamiento de abandonar basura de los demás visitantes (dos Santos 2008; Schultz et al. 2013). Sin embargo, no observamos que la densidad de personas tuviera un efecto sobre la basura abandonada. Es decir que en una misma playa no hubo más basura por visitante los días más concurridos que los días menos concurridos. Esto sugiere que la mayor cantidad de basura por visitante colectada en las playas más grandes podría estar relacionada a otros factores, tales como diferencias en el conjunto de personas que visitan cada tipo de playa. Diversos estudios han mostrado que el hábito de abandonar basura en lugares inapropiados es menos prevalente en personas de mayor edad, y con un mejor nivel socioeconómico y educativo (Santos et al. 2005; Schultz et al. 2011; Slavin et al. 2012; Eastman et al. 2013). En nuestra área de estudio no hemos recopilado información sobre estas características de los visitantes, pero observamos que los más jóvenes se suelen concentrar en las playas más grandes, posiblemente porque concurren en grupos numerosos (Obs. Pers.). Por otro lado, es conocido que distinto tipo de personas tienden a seleccionar distinto tipo de playa (Roig i Munar 2003; Roca \& Villares 2008). Los visitantes que buscan un mayor contacto con la naturaleza tienden a seleccionar playas más tranquilas (Roca \& Villares 2008). Esto podría explicar que los visitantes que buscan playas más pequeñas y por ende con menos cantidad de personas, presenten un comportamiento propenso a cuidar el lugar que visitan y a llevarse su basura, en comparación con aquellos que visitan playas más grandes y con una mayor concurrencia en términos absolutos.

El pedido personalizado para que los visitantes no abandonen basura en las playas no tuvo el efecto esperado. En otros lugares del mundo se ha encontrado que a través de mensajes persuasivos y demostrativos se puede lograr una reducción en la cantidad de personas que abandonan basura en lugares inadecuados (Orams 1997; Cialdini 2003; Brown et al. 2010; Keizer et al. 2008, 2013). Nosotros en cambio no logramos demostrar que con este tipo intervención se pueda disminuir la cantidad de basura abandonada en las playas. Las diferencias entre estudios pueden deberse a razones metodológicas. Mientras que nosotros evaluamos la cantidad de basura acumulada, los estudios mencionados evaluaron el comportamiento individual de las personas en situaciones experimentales mucho más controladas. Es posible que nuestro mensaje haya tenido efectos sobre una cierta proporción de visitantes, pero esto no se haya podido detectar estadísticamente debido a la gran variación en la cantidad de basura que puede dejar una sola persona (Obs. Pers.). El hecho de que en siete de las nueve playas analizadas hayamos encontrado en promedio menos basura cuando se hizo el pedido personalizado que cuando no se hizo, sugiere que esta interpretación puede ser correcta, pero será necesario confirmarla aumentando el esfuerzo de muestreo (y por ende la potencia del análisis estadístico) en futuros estudios.

Por otro lado, también es posible que nuestra estrategia de comunicación no haya sido la adecuada, o que dentro de la población en estudio, las personas que abandonan basura sean poco receptivos a este tipo de mensajes. Dado que en Latinoamérica no existen, a nuestro mejor saber y entender, estudios que hayan evaluado el cambio de comportamiento ante los mensajes persuasivos, es difícil establecer comparaciones con los estudios hechos en otros lugares (ej. Brown et al. 2010 y otros citados allí). Sin embargo, un estudio hecho a lo largo de todas las costas del Pacífico en Chile brinda datos interesantes (Eastman et al. 2013). Según dicho trabajo, un $13 \%$ de las personas admiten abandonar basura en la playa frecuentemente, y otro $31 \%$ admite hacerlo ocasionalmente. La mayor parte de las personas que admiten dejar basura frecuentemente opinaron que las mejores soluciones para el problema consisten en multar a los infractores, o bien tener personal limpiando permanentemente las playas. Esto contrastó con las respuestas de las personas que declararon no abandonar basura nunca (el restante $54 \%$ de los encuestados), quienes consideraron en primer lugar a la educación ambiental como estrategia para solucionar el problema. Estos resultados sugieren que al menos cierta proporción de las personas que abandonan basura no serían sensibles a la educación o interpretación ambiental como modo de solucionar el problema. Otro estudio, hecho en Brasil (Santos et al. 2005), indica que aproximadamente la mitad de las personas que admiten haber abandonado basura en la playa no se sienten culpables por ello. También esto sugiere que dichas personas no serían muy sensibles a los mensajes persuasivos. Dado que nuestra área de estudio es un área protegida, con cartelería que pueden ver todos los visitantes (tanto aquellos que recibieron el pedido personalizado y el 
ejemplo, como aquellos que no) es posible que las personas sensibles a los mensajes tengan siempre el comportamiento adecuado, mientras que las personas insensibles a los mensajes no tengan el comportamiento adecuado, independientemente de nuestra intervención.

En síntesis, nuestro estudio muestra que la reducción de la contaminación por basura en un área protegida no es una tarea sencilla, y que tal vez no se pueda resolver solo con herramientas de la interpretación ambiental. Para avanzar en la solución de este problema será necesario un mayor esfuerzo de comunicación, y tal vez la aplicación de sanciones. A su vez, en el caso de la cuenca en la que se enmarca la reserva bajo estudio, abordar la problemática de degradación ambiental que sufre requiere mayores acciones de coordinación y cooperación entre los actores involucrados en su manejo y preservación (Berardo 2014). Por lo tanto, se deberán realizar acciones de largo plazo en la que se vean involucrados distintos niveles gubernamentales, institucionales y sociales, para lograr modificar los patrones de conducta de aquellos grupos de turistas que no toman en cuenta la magnitud del impacto que genera la contaminación por basura (Marion \& Reid 2007).

Agradecimientos: a las autoridades de la comuna de Cuesta Blanca y a su guardaparque M Sosa, por el apoyo logístico para realizar el estudio, y al guardaparque J Piedrabuena por impulsar las técnicas de comunicación persuasiva en Cuesta Blanca. Asimismo, agradecemos a los vecinos y guardambientes voluntarios de Cuesta Blanca, siempre dispuestos a colaborar con la conservación del área. A R Berardo, MSemmartín y dos revisores anónimos por la lectura crítica del manuscrito y valiosos aportes para mejorarlo.

\section{BIBLIOGRAFÍA}

Abell, R; JB Allan \& B Lehner. 2007. Unlocking the potential of protected areas for freshwaters. Biol. Conserv., 134:48-63.

Ballance, A; PG Ryan \& JK Turpie. 2000. How much is a clean beach worth? The impact of litter on beach users in the Cape Peninsula, South Africa. S. Afr. J. Sci., 96: 210-213

Barragán Muñoz, JM; JR Dadón; SD Matteucci; JH Morello; C Baxendale \& A Rodirguez. 2003. Preliminary basis for an integrated management program for the coastal zone of Argentina. Coast Manage., 31:55-77.

BERARDO, R. 2014. The evolution of self-organizing communication networks in high-risk social-ecological systems. International Journal of the Commons, 8:236-258.

Bravo, M.; MA Gallardo; G Luna-Jorquera; P Núñez; N VÁsquez \& M ThIEL. 2009. Anthropogenic debris on beaches in the SE Pacific (Chile): Results from a national survey supported by volunteers. Marine Pollution
Bulletin, 58:1718-1726.

Brown, TJ; SH Ham \& M Hughes. 2010. Picking up litter: an application of theory-based communication to influence tourist behaviour in protected areas. J. Sustain. Tour., 18:879-900.

CiALDINI, RB. 2003. Crafting normative messages to protect the environment. Curr. Dir. Psicol. Sci., 12:105-109.

Cingolani, AM. 2013. Reserva Ecológica y Recreativa Cuesta Blanca. Boletín Informativo $\mathrm{N}^{\mathrm{O}} 75$. Ecosistemas Argentinos. http://www.ecosistemasarg.org.ar/

Cuesta Blanca. 2011. Folleto sobre normas de comportamiento ambiental. vecinoscuestablanca.blo gspot.com.ar

DADON, JR. 2002. El impacto del turismo sobre los recursos naturales costeros en la costa pampeana. Pp. 101-121 en: JE Dadon \& SD Matteucci (eds.). Zona Costera de la Pampa Argentina. Buenos Aires.

dos Santos, CS; JR dos Santos; RK Oliveira Santana; IS Sobral Oliveira \& LJ Gomes. 2008. Residuos sólidos produzidos por visitantes no Parque Nacional Serra de Itabaiana, Sergipe. Biol. Geral Exper., 2:18-20.

Duncan, GS \& SR Martin. 2002. Comparing the effectiveness of interpretative and sanction messages for influencing wilderness visitors' intended behavior. International Journal of Wilderness, 8:20-25.

Eastman, LB; P Núñez; B Crettier \& M Thiel. 2013. Identification of self-reported user behavior, education level, and preferences to reduce littering on beaches: A survey from the SE Pacific. Ocean Coast Manage., 78: 18-24.

Giorgis MA; AM Cingolani; F Chiarini; J Chiapella; G BArboza; L Ariza Espinar et al. 2011. Composición florística del Bosque Chaqueño Serrano de la provincia de Córdoba, Argentina. Kurtziana, 36:9-43.

HAм, SH. 1992. Environmental Interpretation: A Practical Guide for People with Big Ideas and Small Budgets. Golden, CO: North American.

JUNIPER, T. 2013. What has nature ever done for us? How money really does grow on trees. Profile Books, London, UK.

KARR,JR. 1991. Biological integrity: a long-neglected aspect of water resource management. Ecol. Appl., 1:66-84.

Keizer, K ; S Lindenberg \& L Steg. 2008. The spreading of disorder. Science, 322:1681-1685.

Keizer, K ; S Lindenberg \& L Steg. 2013. The importance of demonstratively restoring order. PloS one, 8:e65137.

Marion, JL \& SE ReID. 2007. Minimising visitor impacts to protected areas: the efficacy of low impact education programmes. J. Sustain. Tour., 15:5-27.

Mayer, PM; SK Reynolds, JR; MD McCutchen \& TJ CANFIELD. 2007. Meta-Analysis of Nitrogen Removal in Riparian Buffers. J. Environ. Qual., 36:1172-1180.

McKInNEY, ML. 2002. Urbanization, biodiversity, and conservation. BioScience, 52:883-890.

Millennium Ecosystem Assessment. 2005. Ecosystems and human well-being. Current state and trends-findings of the condition and trends working group. Island Press, Washington, DC.

Morley SA \& JR Karr. 2002. Assessing and Restoring the Health of Urban Streams in the Puget Sound Basin. Conserv. Biol., 16:1498-1509.

Orams, MB. 1997. The Effectiveness of environmental education: can we turn tourists into 'greenies'? Progress in Tourism and Hospitality Research, 3:295-306.

PÁramo, P. 2010. Aprendizaje situado: creación y modificación de prácticas sociales en el espacio público 
urbano. Psicologia $\mathcal{E}$ Sociedade, 22:130-138.

Roca, E \& M Villares. 2008. Public perceptions for evaluating beach quality in urban and semi-natural environments. Ocean Coast Manage., 51:314-329.

Roig i Munar, FX. 2003. Análisis de la relación entre capacidad de carga física y capacidad de carga perceptual en playas naturales de la Isla de Menorca. Investigaciones Geográficas, 31:107-118.

SAntos, IR; AC Friedrich; M WaLlner-Kersanach \& G FILLMAN. 2005. Influence of socioeconomic characteristics of beach users on litter generation. Ocean Coast Manage., 48:742-752.

Schultz, PW; RJ Bator; L Brown Large; CM Bruni \& JJ TABANICO. 2013. Littering in context: personal and environmental predictors of littering behavior. Environ.
Behav., 45:35-59.

SeCo Pon, JP \& ME BeCheruCCI. 2012. Spatial and temporal variations of urban litter in Mar del Plata, the major coastal city of Argentina. Waste Manage., 32:343-348.

Slavin, C; A Grage \& ML Campbell. 2012. Linking social drivers of marine debris with actual marine debris on beaches. Mar. Pollut. Bull., 64:1580-1588.

Stiva, L. \& D. Willams. 2001. Buffer zone versus whole catchment approaches to studying land use impact on river water quality. Wat. Res., 35:3462-3472.

Thiel, M; M Bravo; IA Hinojosa; G Luna; L Miranda; P Nuñez ET AL. 2011. Anthropogenic litter in the SE Pacific: an overview of the problem and possible solutions. Journal of Integrated Coastal Zone Management, 11:115-134. 